\title{
LA IMAGEN ESTADÍSTICA DE LA EMIGRACIÓN INTERNACIONAL EN ESPAÑA: ANÁLISIS DE LAS NUEVAS FUENTES EN RELACIÓN CON LA FIABILIDAD DE LA ESTADÍSTICA DE VARIACIONES RESIDENCIALES
}

\author{
Carmen Ródenas \\ Mónica Martí \\ Departamento de Análisis Económico Aplicado. \\ Universidad de Alicante \\ crodenas@ua.es,mmart@uua.es
}

\section{RESUMEN}

Dos razones aconsejan retomar el análisis de los números migratorios de las estadísticas españolas. Por un lado, porque desde 2008 el país atraviesa una profunda crisis económica y se desconoce con qué intensidad ha afectado a los flujos de salida de extranjeros desde España al exterior. Y, por otro lado, porque el INE acaba de difundir nuevas fuentes migratorias derivadas de innovadores procedimientos estadísticos. El trabajo analiza los resultados de estas fuentes en relación con las migraciones internacionales, utilizándolos para evaluar el grado de subestimación de la emigración exterior de la, ya tradicional, Estadística de Variaciones Residenciales. Adicionalmente, en el trabajo se han obtenido otros dos resultados que, desde el punto de vista de la calidad de las nuevas fuentes estadísticas, deberían ser objeto de reflexión.

Palabras clave: migraciones internacionales, fuentes estadísticas, emigración, censo de población, encuestas demográficas.

Fecha de recepción: enero 2015.

Fecha de aceptación: octubre 2015. 


\section{ABSTRACT}

There are two good reasons for taking another look at the analysis of migration figures from the Spanish statistics. Firstly, because since 2008 the country has been undergoing a profound economic crisis and the extent to which it is impacting on the outflows of foreigners from Spain is unknown. And, secondly, because the INE has just made new sources derived from innovative statistical procedures available. The work has focused on analyzing the international migration figures of these sources and uses them to evaluate the degree of underestimation of the international migration outflows in the traditional Residential Variation Statistics. Furthermore, two additional conclusions have been reached in the paper that, from the point of view of the quality of the new statistical sources, should be reflected upon.

Keywords: international migration, statistical sources, emigration, census, demographic surveys.

\section{INTRODUCCIÓN}

España, en comparación con el resto de países de la UE, recibió uno de los más intensos contingentes migratorios internacionales en los años previos a la recesión económica, entre 2000 y 2007. Este fenómeno, claramente percibido por la sociedad y los medios de comunicación, no se reflejó de similar modo por las fuentes estadísticas nacionales disponibles en aquellos momentos. Por diferentes motivos de carácter metodológico, el fuerte crecimiento en el ritmo de las entradas de extranjeros desde 1999 no se registró adecuadamente en la Encuesta de Migraciones tributaria de la Encuesta de Población Activa (EPA) o en la ocasional Encuesta Nacional de Inmigrantes (ENI) de 2007 ni, tampoco, en 2001 por el Censo de Población ${ }^{1}$. La única fuente que reflejó el enérgico aumento de las llegadas fue la Estadística de Variaciones Residenciales (EVR), registro administrativo en el que figuran las altas (y bajas) por cambio de residencia declaradas por la población que habita en España, con el que se actualiza tanto la Base Padronal centralizada por el Instituto Nacional de Estadística (INE) como los correspondientes Padrones Municipales de población.

Esta estadística, que se inicia en 1961 y tiene una gran tradición en el INE, ha experimentado importantes mejoras a lo largo del tiempo, ampliando su cobertura e incorporando procedimientos informáticos que han mejorado extraordinariamente su calidad (INE, 2014a). La EVR se asienta en la obligación de los residentes en España de registrar ante las autoridades locales cualquier cambio de residencia habitual, aunque en determinadas circunstancias son los ayuntamientos quienes de oficio registran esos cambios. Hasta 2001 se generaba a partir de la agregación de los ficheros de variaciones residenciales que mensualmente enviaban los municipios al INE. A partir de entonces se obtiene directamente de la Base Padronal del INE que centraliza y depura los Padrones Municipales, con lo que la calidad de la EVR ha

1 Estas cuestiones se explican en Ródenas y Martí (1997) y Martí y Ródenas $(2004,2007)$ para la Encuesta de Migraciones/EPA; para la ENI en los de Martí y Ródenas (2012a) y Ródenas y Martí (2013), y en relación con el Censo de 2001 en Ródenas y Martí (2009a y b). 
mejorado sustancialmente. Según el INE (2014b), actualmente el proceso de elaboración de la estadística tiene establecidos controles para detectar y corregir los errores (encontrados en torno al $0,18 \%$ de los registros) y, con el fin de garantizar que las variaciones correspondan realmente a cambios de residencia, no se contabilizan aquellas bajas al exterior para las cuales se detecta que el habitante tiene un alta posterior en el mismo año.

El hecho de que la inscripción en el Padrón se exija como condición necesaria para el ejercicio de derechos muy básicos, como el de cobertura sanitaria, el de escolarización o para la obtención de permisos legales de residencia, hace que las altas por variación residencial sean consideradas buen reflejo del flujo de la inmigración procedente del extranjero ${ }^{2}$. Sin embargo, la observación directa de las variaciones por baja internacional en el Padrón (disponibles desde 2002), pudiera ser insuficiente para la medición correcta de los flujos migratorios de salida al exterior. En especial, en el caso de los extranjeros, ya que son pocos los que ordenan su baja en el Padrón en el momento de su marcha de España. De hecho, para algunos autores, en comparación con la inmigración, la imagen estadística de la emigración al exterior, tanto de nacionales como de extranjeros, se caracteriza por su borrosidad, siendo el cómputo de las bajas de escasa cobertura. Incluso, como recuerdan Domingo y Sabater (2013), De Castro (2004) y Garrido (2004), con anterioridad a la crisis existía esa deficiente calidad debido, entre otras cosas, a que prevalecía un sistema de control exclusivamente municipal, y tanto los municipios eran reacios a perder población como había migrantes que por diversos motivos querían mantener su inscripción padronal pese a abandonar el país. Frente a estas situaciones, en la actualidad el INE (2014c) señala que el propio mecanismo de bajas efectuadas de oficio por los ayuntamientos sobre aquellos casos de no residencia habitual en el municipio garantizan que la emigración exterior de personas de nacionalidad extranjera acabe, en gran medida, registrándose en el Padrón, tarde o temprano.

Ante este panorama, dos son las razones que aconsejan retomar ahora el análisis de los números migratorios de las estadísticas españolas. Por un lado, porque desde 2008 la economía española atraviesa una profunda crisis económica y no conocemos con certeza cómo se ha modificado el patrón migratorio. Dicho de otro modo, no sabemos cuántos inmigrantes se han marchado y cuántos quedan de los que llegaron antes de la recesión. Y, por otro lado, porque, junto a la publicación en diciembre de 2013 de los resultados migratorios detallados del Censo de Población de 2011 elaborado por el INE sobre la base de una nueva metodología, se dispone de nuevas fuentes estadísticas oficiales. En abril de 2014 el INE difundió el primer año, 2013, de la Encuesta Continua a los Hogares (ECH); la serie de la Estadística de Movilidad Laboral y Geográfica (EMLG) vio la luz en noviembre de 2013 y, finalmente, en este mismo año comienza a ser divulgada la Estadística de Migraciones (ESMI). Habida cuenta, además, de que se trata de fuentes derivadas de procedimientos estadísticos puestos en marcha por primera vez, el trabajo es todavía más relevante.

2 INE (2014c), Domingo y Sabater (2013), Poulain, Perrin y Singleton (2006:122 y 212). No obstante, existe la sospecha de que en ciertos periodos -anteriores a la crisis- se haya producido una ocasional sobreestimación de la inmigración al registrarse altas sin correspondencia con verdaderos movimientos migratorios, precisamente para el disfrute de los anteriores derechos básicos, como también se hacen eco INE (2009:141), Domingo y Sabater (2013:8 y ss.), Ródenas y Martí (2006, 2009a y c), Martí y Ródenas (2012b) y Susino (2011 y 2012). 
Tabla 1

RESUMEN DE LAS NUEVAS FUENTES ESTADÍSTICAS

\begin{tabular}{|c|c|c|c|}
\hline Fuente & Acrónimo & $\begin{array}{r}\text { Objetivo } \\
\end{array}$ & $\begin{array}{c}\text { Características } \\
\end{array}$ \\
\hline $\begin{array}{l}\text { Encuesta } \\
\text { Continua a los } \\
\text { Hogares }\end{array}$ & $\mathrm{ECH}$ & $\begin{array}{l}\text { Convertirse en la base de } \\
\text { las operaciones estadísticas } \\
\text { que requieran la extracción } \\
\text { de submuestras de viviendas } \\
\text { principales para otras en- } \\
\text { cuestas a hogares. }\end{array}$ & $\begin{array}{l}\text { La muestra es encuestada a lo lar- } \\
\text { go de los cuatro trimestres y se } \\
\text { ofrece información anual sobre las } \\
\text { características demográficas de la } \\
\text { población, de los hogares y de las } \\
\text { viviendas, similar a la censal. }\end{array}$ \\
\hline $\begin{array}{l}\text { Estadística } \\
\text { de Movilidad } \\
\text { Laboral y } \\
\text { Geográfica }\end{array}$ & EMLG & $\begin{array}{l}\text { Investigar la relación entre la } \\
\text { situación en el mercado labo- } \\
\text { ral y los cambios de residen- } \\
\text { cia de las personas. }\end{array}$ & $\begin{array}{l}\text { Integra los datos individuales de la } \\
\text { EPA con la información individual } \\
\text { registrada en la Base Padronal del } \\
\text { INE. }\end{array}$ \\
\hline $\begin{array}{l}\text { Estadística de } \\
\text { Migraciones }\end{array}$ & ESMI & $\begin{array}{l}\text { Mejorar la observación del } \\
\text { fenómeno migratorio más } \\
\text { allá de las altas y bajas por } \\
\text { variación residencial, cen- } \\
\text { trándolo en los verdaderos } \\
\text { movimientos migratorios. }\end{array}$ & $\begin{array}{l}\text { Sobre la base de la EVR, se estima } \\
\text { la fecha de ocurrencia de las emi- } \\
\text { graciones al extranjero de oficio, se } \\
\text { intenta ajustar el desfase temporal } \\
\text { entre la ocurrencia del movimien- } \\
\text { to y el momento de su registro, se } \\
\text { eliminan las bajas y altas consecu- } \\
\text { tivas con menos de doce meses de } \\
\text { diferencia y se imputa el país de } \\
\text { nacimiento, de nacionalidad y de } \\
\text { origen o destino en los casos que } \\
\text { no consta. }\end{array}$ \\
\hline
\end{tabular}

Fuente: elaboración propia.

Así, a grandes rasgos, el Censo de 2011 no se ha levantado como un censo clásico con la enumeración exhaustiva de las viviendas, sino que ha combinado la información de diferentes registros administrativos (entre ellos, el Padrón) con una encuesta específica a unos tres millones de viviendas (12,3\% de la población). Por su parte, la ECH ha sido diseñada para convertirse en la espina dorsal de las operaciones estadísticas que requieran la extracción de submuestras de viviendas principales para otras encuestas dirigidas a hogares en España. La muestra de la ECH se encuesta a lo largo de los cuatro trimestres y con ella se ofrece información anual sobre las características demográficas básicas de la población, de los hogares y de las viviendas, similar a la censal pero con frecuencia mayor que la decenal. El caso de la EMLG es diferente. Su innovación es la integración de los datos proporcionados por la muestra EPA con la información correspondiente individual registrada en la Base Padronal del INE, de modo que investiga la relación entre la situación en el mercado laboral de las personas y su historial de cambios de residencia. La última fuente, la ESMI, es fruto de la explotación estadística de la EVR y su fin es mejorar la observación del fenómeno demográfico más allá de las simples declaraciones de altas y bajas, intentando centrarlo en aquellas asociadas con verdaderos movimientos migratorios. Así, en la ESMI, los técnicos del INE 
estiman la fecha de ocurrencia de las emigraciones al extranjero ejecutadas de oficio por los ayuntamientos; intentan ajustar el desfase temporal entre la ocurrencia del movimiento migratorio y el momento de su registro; reconducen la información padronal al concepto de migración eliminando bajas y altas consecutivas con menos de doce meses de diferencia protagonizadas por un mismo individuo; y, finalmente, imputan el país de nacimiento, de nacionalidad, de origen o destino en los casos en que no constan.

Por tanto, el trabajo se centra, primero, en analizar los resultados que arrojan estas fuentes sobre las migraciones internacionales recientes en España; indagar si dibujan un panorama similar, y si no lo hacen explicar por qué. Y, en segundo lugar y utilizando las nuevas fuentes, en intentar precisar el grado de subestimación (sobreestimación) de las bajas (altas) de la EVR, es decir, evaluar su fiabilidad. Este último objetivo se justifica porque la inclusión en la EVR, a diferencia de los países de nuestro entorno, de todas las personas que residen en el territorio de forma legal o no, proporciona a esta estadística una enorme ventaja a la hora describir adecuadamente la intensidad y los cambios en el fenómeno migratorio.

Para ello, el trabajo se estructura de la siguiente manera. En el apartado primero se presenta la evolución comparada de la inmigración procedente del exterior desde los años sesenta según las diferentes fuentes censales, registrales y las generadas mediante encuestas. En el segundo apartado se analiza la coherencia de la información migratoria entre los sucesivos censos de población. Una vez verificado que el Censo de 2011 no tiene los problemas de subestimación de la inmigración que presentaba el Censo de 2001, se utiliza para realizar una primera aproximación de la subestimación en la EVR de las bajas al exterior de los nacidos fuera para el período 2002-2011. En el tercer apartado se desarrolla el análisis de las nuevas fuentes estadísticas -ECH, EMLG y ESMI- y se utilizan como base para analizar la subestimación de las bajas de la EVR a través de diferentes ejercicios de verificación de las ecuaciones de balance demográfico. En el último apartado se resumen las principales conclusiones y, finalmente, el trabajo se cierra con el listado de las referencias citadas.

\section{UNA COMPARACIÓN DE LA INMIGRACIÓN EXTERIOR SEGÚN LAS DIFERENTES FUENTES}

La figura 1 recoge de modo gráfico los números migratorios de las estadísticas oficiales españolas. Puede apreciarse que censos, encuestas y registros exhiben diferentes relatos del mismo fenómeno: la inmigración procedente del exterior. Tanto los ritmos de entrada como los niveles a los que se mueven las series difieren de forma considerable. Parte de esas diferencias, en concreto las que existen entre la EVR o la ESMI (fuentes registrales) respecto del resto, deben atribuirse a que las dos mencionadas cuentan movimientos (migraciones) en el momento en que se declaran, mientras que los censos y las encuestas estiman personas (migrantes); esto es, inmigrantes supervivientes ${ }^{3}$ en el momento en que se levantan estas fuentes. Tampoco hay que olvidar que para comparar correctamente los supervivientes por año de entrada, en la EVR y en la ESMI de la figura 1 debería descontarse de las altas por inmigración de cada año $t$, las bajas por emigración al exterior correspondientes a esa cohorte

3 Se trata de los inmigrantes que no han fallecido y que permanecen en el país todavía en el momento del recuento. 
Figura 1

INMIGRACIÓN ANUAL PROCEDENTE DEL EXTERIOR (INMIGRANTES E INMIGRACIONES)

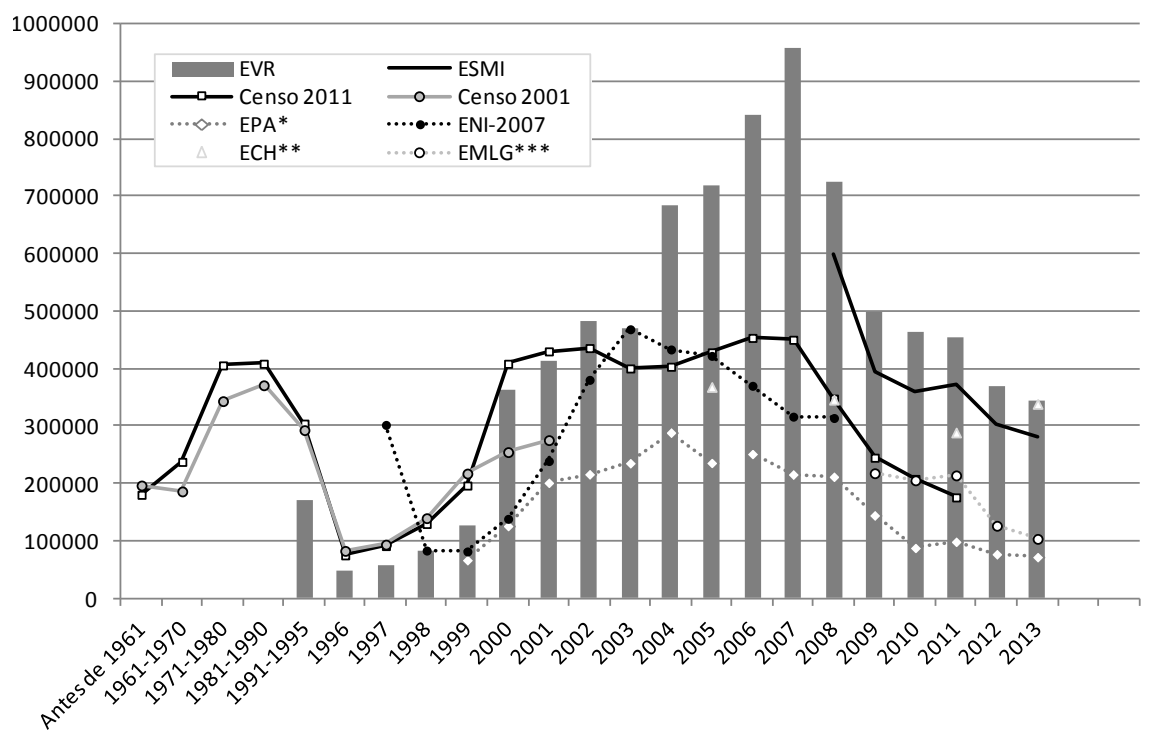

NOTAS: *EPA: Entre 1999 y 2005 se trata de la Encuesta de Migraciones/EPA; **ECH: datos provisionales a 1/7/2013. Año 2005 (media periodo 2002-2006), año 2008 (media periodo 2007-2008) y año 2011 (media periodo 2010-2011); ***EMLG: solo mayores de 15 años.

Fuente: INE (EVR, ESMI, EPA, ENI, ECH, EMLG, Censos de Población de 2001 y 2011) y elaboración propia.

de entrada en $t$, independientemente de cuándo se produzcan. Aunque el INE posiblemente sí dispone de tal información (solo se trata de un enlace de registros de altas y bajas en la Base Padronal) no lo difunde ${ }^{4}$.

Teniendo esto en cuenta, no hay duda de que, teóricamente, las cifras de inmigraciones brutas deberían estar por encima de las de inmigrantes, por las razones siguientes: no se han descontado de las entradas las salidas al exterior; cada migrante puede tener más de un movimiento posterior a su entrada y, finalmente, porque los inmigrantes tienen que sobrevivir hasta el momento posterior en el que se cumplimenta el cuestionario del censo o encuesta. El hecho de que hasta 2001 esto no se produzca siempre señaliza que, efectivamente, la EVR estaba subestimando el flujo de entradas internacionales. Afortunadamente, a partir de este año mejora la cobertura de la EVR tanto porque el proceso estadístico se depura extraordinariamente con la Base Padronal centralizada del INE, como por la progresiva relevancia (para los procesos de regularización, el disfrute de derechos básicos...) que el empadronamiento tiene para los inmigrantes.

4 Por eso, en la figura 1 hemos optado por mostrar solo el flujo de inmigración de la EVR y de la ESMI. No sería correcto descontar los flujos anuales de emigración (de llegadas a lo largo de todos los años anteriores) de los de entradas de cada año, si lo que interesa es comparar entre las fuentes el ritmo de llegadas y la permanencia de las cohortes de entrada en España. 
El perfil de las entradas de la EVR a partir de 2001 se muestra muy ajustado a las fases que atraviesa el ciclo económico español. Creciendo hasta 2007 y disminuyendo desde la irrupción de la crisis en 2008 (quizá, de un modo no tan abrupto como podría esperarse). No es muy convincente, por el contrario, el perfil temporal de la ENI-2007 (con un máximo de inmigrantes llegados en 2003) ni el que dibuja la EPA (que sitúa su máximo un año más tarde, en 2004). De hecho, esas figuras pueden explicarse a través de los problemas metodológicos que presentan esas dos encuestas para capturar a los inmigrantes 5 .

El número de inmigrantes registrados por los consecutivos censos de población tampoco se comporta del modo esperado. El Censo de 2001 se encuentra a un nivel, por lo general, inferior al del posterior de 2011. En especial, para los años 2000 y 2001. En trabajos anteriores ya se advertía sobre la infravaloración en el Censo de 2001 de la inmigración acaecida en los años más próximos al del levantamiento del censo; por eso, y con la intención de utilizar el Censo de 2011 para valorar la EVR, comenzaremos por analizar las fuentes censales con detenimiento.

\section{LA INFORMACIÓN CENSAL SOBRE LA MOVILIDAD DE LA POBLACIÓN}

\section{III.1. La coherencia entre las fuentes censales y registrales}

En la figura 2 se muestra cómo deberían relacionarse teóricamente los perfiles temporales de registros y censos de población. Ya se ha dicho que los registros miden altas en el momento de la llegada/declaración, por lo que sus cifras deben estar por encima de las censales que recogen inmigrantes supervivientes. Nuevamente por razones de supervivencia (por mortalidad o emigración), las series censal y registral, además, deberían reducir su distancia a medida que se aproximan al año del levantamiento del censo. Al mismo tiempo, dos censos consecutivos levantados en los años $t$ y $t+10$ que ofrezcan datos de inmigrantes para el mismo periodo, por ejemplo entre $t$ - 10 y $t$, deberían mostrar una senda de llegadas similar en la que, además, los niveles del censo de $t$ deberían situarse por encima de los de $t+10$.

Sin embargo, en la figura 1 ya se apreció que esto no sucede con nuestros datos. Se sabía que el Censo de 2001 presentaba problemas graves para captar el año de llegada de los inmigrantes. Aparentemente, ante la falta de respuesta parcial a las preguntas migratorias en el cuestionario censal, el procedimiento de imputación seguido por el INE tenía como consecuencia, entre otras, la subestimación de la inmigración producida recientemente ${ }^{6}$. Esto es lo que se puede ver en la figura 1 al comparar el Censo de 2001 con la EVR, pero ya no cabe

$5 \quad$ Ver nota 1.

6 Esta falta de respuesta era la consecuencia de solicitar a todos los encuestados a declarar el año desde el que residían (aunque fuera desde que nacieron) en el municipio, indicando el anterior lugar de residencia si es que antes fue otro. Si el encuestado no contestaba pero su lugar de nacimiento y de residencia en 2001 no coincidían, evidentemente se trataba de un inmigrante no auto-declarado cuyo cuestionario censal bien debía ser depurado por incompleto o bien debía serle imputado un año de llegada. Para Ródenas y Martí (2009a y b) el procedimiento de imputación (reconocido por el INE que sí existió) bien pudo ser equivalente a asignar la fecha de nacimiento como fecha de llegada. Este proceder lanzaría hacia el pasado el año de llegada de los inmigrantes exteriores que acceden a España al inicio de la década del 2000, haciendo que su movilidad se registrara anteriormente. Y lo cierto es que mientras que el Censo de 1991 elevaba a 325.931 los extranjeros llegados a España antes de 1991, el Censo de 2001 sitúa esta cifra en 467.633; esto es, más de cien mil personas por encima. Por eso, cuando en el 2000 empiezan a crecer las entradas, un sistema de imputación como el descrito tendía a infravalorar la inmigración más reciente. 


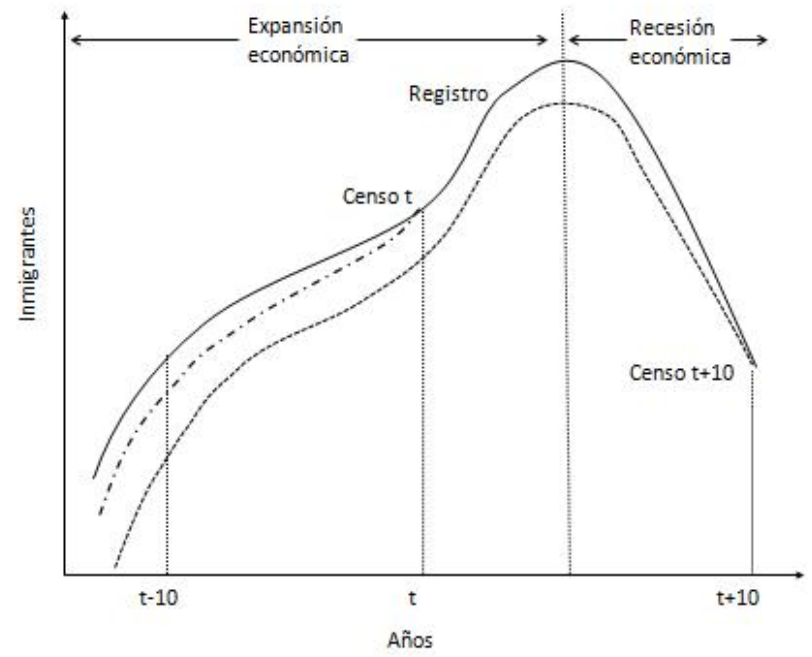

Fuente: elaboración propia.

duda alguna al analizar las magnitudes migratorias reveladas por el nuevo Censo de 2011. Los llegados a España procedentes del extranjero en 2000 y 2001 son estimados en poco más de 530.000 personas por el Censo de 2001. Sin embargo, diez años más tarde y con el Censo de 2011, resulta que el mismo colectivo ha superado las 839.000 personas $^{7}$; en este caso, una diferencia de más de 300.000 inmigrantes tan sólo para estos dos años 2000 y 2001.

Este mejor ajuste en la captura de los inmigrantes exteriores es muy posible que se deba a los numerosos cambios en la metodología seguida por el INE para elaborar el último censo ${ }^{8}$. Primero, porque al emplear un método mixto basado en registros administrativos que se combinan con una encuesta por muestreo a la población, disminuye el peso del trabajo de campo. Esto permite trabajar con una organización más reducida y mejorar la formación y control de los agentes censales, contribuyendo así a reducir errores de medida y de cobertura. De hecho, en el caso de los extranjeros ello ha permitido que se haya tenido un exquisito cuidado en la verificación de su residencia en España, lo que ha conducido a una mejor estimación de sus factores de recuento en el Fichero Precensal en función de los resultados de la encuesta y, en definitiva, un mejor cálculo de su elevación al total.

En segundo lugar, porque aunque el principal canal para la recogida de la información censal haya sido el del correo convencional (el $51 \%$ de los cuestionarios han sido remitidos en papel), un $39 \%$ de los hogares ha cumplimentado el cuestionario a través de Internet, a diferencia del Censo de 2001 en el que sólo lo hicieron 13.768 hogares $^{9}$, un $0,09 \%$ del

7 La variable utilizada es el año de llegada a España (ANOE) de todos los procedentes del extranjero en ambos censos.

8 Para cuestiones de trabajo de campo, calidad y fiabilidad del Censo de 2011 puede consultarse INE (2014d, e).

$9 \operatorname{INE}(2014 \mathrm{f})$. 
total. La gran ventaja de esta vía es que la aplicación informática contempló controles de ruta, rango y consistencia entre las distintas preguntas, lo que tuvo que reducir radicalmente la falta de respuesta. Es lo mismo que sucedió cuando los agentes censales recogieron la información in situ (aproximadamente en el 10\% de los hogares entrevistados): se utilizaron tablets con aplicaciones que también impedían la introducción de datos inválidos y controlaban las inconsistencias, incompatibilidad o incongruencia entre las respuestas.

En tercer lugar, porque si trabajar con una muestra en lugar de con toda la población y utilizar intensamente los filtros que proporciona la tecnología ya ha debido reducir la falta de respuesta en el último Censo, las modificaciones realizadas en el propio cuestionario ${ }^{10}$ también han contribuido a su disminución. Por un lado, porque se ha realizado un acertado cambio en el orden en que se efectúan las preguntas de lugar/fecha de nacimiento y nacionalidad, y las referentes a las migraciones. Mientras que en el Censo de 2001 las primeras se respondían en un impreso de datos padronales y las segundas en un cuestionario -en hoja aparte- de hogar, en el Censo de 2011 aparecen todas ellas en un único cuestionario (el individual) y en orden consecutivo. Esta nueva presentación predispone a los sujetos a responder de forma consistente y a no incurrir accidentalmente en falta de respuesta parcial, a diferencia de lo que sucedía con el Censo de 2001. En segundo lugar, porque la formulación de las preguntas migratorias es mucho más clara en el último Censo. En efecto, la nueva pregunta acerca del año de llegada a la actual residencia ahora ofrece al individuo la opción de señalar que permanece en ella desde su nacimiento, caso en el que ya no se le hace ninguna pregunta adicional sobre su movilidad. La ventaja es que permite que el propio individuo se declare migrante o no. Lo que sucedía en el Censo de 2001 es que se continuaba (innecesariamente) preguntando a estas personas por su año de llegada a España y por su residencia hace diez años. Y, a la vista de las respuestas era el INE, no el individuo, quien decidía si se trataba de un inmigrante o no. El problema es que este procedimiento tenía más posibilidades de que los individuos incurrieran en contradicción y el INE se viera obligando a hacer imputaciones.

Por último, indica el INE que los errores ajenos al muestreo, como la falta de respuesta total o parcial y la existencia de algunos duplicados, que se detectaron finalmente en los cuestionarios del Censo de 2011, se corrigieron utilizando programas específicos diseñados según la naturaleza de cada variable censal. En este contexto, parece bastante lógico suponer que en el caso de falta de respuesta en las cuestiones de movilidad, el año imputado de llegada debería ser preferentemente el mismo que consta en la base padronal, que para eso es el marco poblacional de la encuesta censal.

\section{III.2. Subestimación de las bajas al exterior de la EVR a partir del Censo de 2011}

Si aceptamos que no hay indicios para considerar que el Censo de 2011 subestime la inmigración reciente ${ }^{11}$, entonces y a la vista de la figura 1 , queda otra cuestión por resolver: ¿por qué las cifras de entradas anuales del último Censo y de la EVR -o de la ESMI- no se acercan progresivamente a medida que se aproxima el momento censal? Si la probabilidad

10 Los cuestionarios están disponibles en la página web del INE dedicada a las cifras de población y censos demográficos (http://www.ine.es/inebmenu/mnu_cifraspob.htm).

11 Además de considerar los cambios metodológicos mencionados, se han realizado, además, ejercicios como los que plantean Ródenas y Martí (2009a) para el Censo de 2011, sin encontrar problemas similares a los de 2001. 
de re-emigrar al extranjero o de defunción de los inmigrantes fueran constantes en el tiempo, para los recién llegados ha transcurrido menos lapso para que se produzca la ocurrencia del suceso (emigración o fallecimiento), por lo que las cifras de inmigrantes supervivientes (censo) deberían ser gradualmente similares a las entradas registradas (EVR o ESMI). Sin embargo, la crisis que llevamos arrostrando desde 2008 ha empeorado extraordinariamente las condiciones del mercado laboral conduciendo, teóricamente, a un aumento en la probabilidad de emigrar. Por eso, la diferencia entre el Censo de 2011 y las altas de la EVR bien podría interpretarse como una aproximación a la cuantía total de la re-emigración de los inmigrantes de la oleada de la primera década del 2000. Precisamente, las salidas del país, la parte estadísticamente más borrosa de la EVR.

Tabla 2

SUBESTIMACIÓN DE LAS BAJAS AL EXTERIOR DE LOS NACIDOS FUERA EN LA EVR A PARTIR DEL CENSO DE 2011 (2002-2011)

\begin{tabular}{|c|c|}
\hline Altas 2002-2011 procedentes del exterior (EVR) & 6.070 .476 \\
\hline $\begin{array}{l}\text { Personas llegadas entre 2002-2011 que siguen en España a finales de } \\
2011 \text { (Censo de 2011) }\end{array}$ & -3.381 .628 \\
\hline \multicolumn{1}{|c|}{ Diferencia: } & 2.688 .848 \\
\hline Total bajas 2002-2011 con destino exterior (EVR): 1.613.011 & \\
\hline H $_{\mathbf{0}}$ mínima: restar todas las bajas 2002-2011 & 1.075 .837 \\
\hline Mínimo \% de subestimación de las bajas en la EVR $_{\mathbf{4 0 , 0 1 \%}}^{*}$ \\
\hline H $_{\mathbf{0}}$ máxima: restar el 60\% de las bajas 2002-2011 & 1.721 .042 \\
\hline Máximo \% de subestimación de las bajas en la EVR & $\mathbf{6 4 , 0 1 \%} * *$ \\
\hline
\end{tabular}

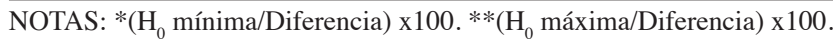

Fuente: INE (EVR y Censo de Población de 2011) y elaboración propia.

Suponiendo, pues, que tanto el Censo de 2011 como las altas de la EVR reflejan correctamente el fenómeno migratorio, entonces el sumatorio de las diferencias anuales entre ambas fuentes en el periodo 2002-2011 podría darnos una estimación de las salidas de los inmigrantes que formaron parte de la reciente ola de entradas en España y, por tanto, facilitar una primera aproximación al grado de la subestimación de las bajas en la EVR. Centrándonos en los inmigrantes nacidos fuera ${ }^{12}$, en la tabla 2 se presenta un primer ejercicio en el que se estima el posible grado de subestimación de las bajas de la EVR.

A partir de la diferencia entre las altas registradas por la EVR entre 2002 y 2011 de inmigrantes nacidos fuera y los inmigrantes nacidos fuera censados en 2011 que entraron en España entre 2002 y 2011 , se ha obtenido una diferencia que representa ${ }^{13}$ fundamentalmente las bajas por salida al exterior y, en mucho menor grado, podría recoger las migraciones múltiples de una misma persona procedente del extranjero. Mediante las bajas consignadas anualmente en la EVR

12 El uso de la variable nacionalidad podría conducir a errores ya que el volumen de nacionalizaciones de extranjeros entre 2002 y 2013 asciende a 1.042 .613 personas.

13 Se ha descartado descontar las defunciones. En primer lugar porque en los datos del Movimiento Natural de la Población Española (MNPE) del INE solo se dispone de los decesos de los extranjeros, no de los nacidos fuera. $\mathrm{Y}$, en segundo lugar, porque tampoco se conoce el año de llegada a España de esos extranjeros. 
se sabe el total registrado de la emigración al exterior para el periodo 2002-2011, de modo que sustrayendo su cuantía de la diferencia anterior se podría aproximar el grado de subestimación de las salidas en la EVR. El problema es que no conocemos cuántas de esas bajas corresponden a altas (entradas previas) habidas en ese mismo periodo. Por eso, en la tabla se plantean dos sencillas hipótesis para descontarlas, que dan lugar a una primera horquilla en la que podría situarse la subestimación. Por un lado, si la totalidad de las bajas se atribuye a entradas exclusivamente entre 2002 y 2011 ( $\mathrm{H}_{0}$ mínima), la EVR dejaría de tener registrado el 40,01\% de las bajas. La banda superior de la horquilla ( $\mathrm{H}_{0}$ máxima) da una subestimación del 64,01\%, que se construye a partir de atribuir al periodo tan sólo el $60 \%$ de las bajas registradas, que es la proporción de los inmigrantes procedentes del extranjero que llegaron según el Censo entre 2002 y 2011.

\section{IV. ¿QUÉ APORTAN LAS NUEVAS FUENTES ESTADÍSTICAS SOBRE LA CALIDAD DE LA EVR?}

\section{IV.1. La Encuesta Continua de Hogares (ECH)}

Esta encuesta tiene como población objetivo la población y los hogares, residentes en España, en cada una de sus comunidades autónomas y en cada una de las provincias. Inicialmente se centra sólo en los residentes en viviendas familiares. Como se explica en INE (2014g), se genera a partir de entrevistas a lo largo de los cuatro trimestres y se ofrecerá anualmente los datos provisionales (con fecha de referencia a 1 de julio de cada año $t$ a partir de los cuatro ficheros trimestrales del año $t$ ) y los resultados definitivos (con fecha de referencia a 1 de enero de cada año $t$ a partir de los cuatro ficheros trimestrales del año $t-1$ y los otros cuatro del año $t$ ). La idea es utilizar esta encuesta, además, como el marco de viviendas principales para cualquier otra encuesta dirigida a los hogares.

El marco muestral de la ECH es el conjunto de huecos (direcciones postales desagregadas hasta el nivel de planta y puerta dentro de un edificio) proporcionado por el Censo de Edificios de 2011. Para la selección de la muestra se utiliza un muestreo bietápico estratificado, en el que las unidades de primera etapa son las secciones censales y las de segunda etapa son los huecos existentes en la sección después del recorrido censal. Se investiga al año un total de 1.600 secciones censales y 65 huecos dentro de cada sección censal, lo que hace un tamaño de muestra anual de 104.000 unidades investigadas (huecos o viviendas), aproximadamente unas 25.000 viviendas al trimestre.

Cada año se renueva la mitad de la muestra de secciones (unas 800, con ritmo de unas 200 de las 400 secciones que se visitan cada trimestre) y en las secciones que permanecen en la muestra, se actualizan las direcciones y se renueva toda la muestra de huecos. Por tanto, cada sección permanece en la muestra durante dos años consecutivos, pero cada hueco solo forma parte de la muestra una vez. Esto supone que no hay muestra común de huecos entre dos entrevistas. Cuando se producen incidencias en los huecos -hueco inaccesible; vivienda seleccionada anteriormente (hace menos de tres años); negativa; ausencia; incapacidad para contestar; no encuestable (el hueco seleccionado es un local comercial, una vivienda colectiva o un hueco destinado a actividades profesionales); duplicado (hay al menos otro hueco en la muestra con la misma dirección postal); hueco ilocalizable; no contactado o vacío (vivienda sin residentes)-, no hay muestra de reserva o de sustitución pues todos los huecos de la muestra se consideran titulares. 
Finalmente, las estimaciones relativas a las características de población (sexo, edad, año de nacimiento, nacionalidad y lugar de nacimiento) se obtienen utilizando estimadores calibrados. Las variables de calibrado serán las Cifras de Población estimadas por el INE a 1 de enero y a 1 de julio de cada año, según se trate de estimaciones definitivas o provisionales.

\section{IV.1.1. Subestimación de las bajas al exterior de los nacidos fuera en la EVR a partir de la ECH}

En la tabla 3 mostramos los resultado de un ejercicio similar al realizado con el Censo de 2011 , pero con la ECH como la estadística de comparación y tomando una fecha de referencia más actual, el 1 de julio de 2013. Para ello, las altas y bajas de la EVR se han ajustado a los periodos anuales marcados por la ECH y, también, en la $\mathrm{H}_{0}$ máxima se ha modificado el porcentaje de bajas asignables al periodo $(65,3 \%)$ de acuerdo con la distribución anual de la entrada de inmigrantes de la ECH.

Tabla 3

SUBESTIMACIÓN DE LAS BAJAS AL EXTERIOR DE LOS NACIDOS FUERA EN LA EVR A PARTIR DE LAECH (JULIO 2002 - JUNIO 2013)

\begin{tabular}{|c|c|}
\hline Altas 2002-2013 procedentes del exterior (EVR) & 6.341 .480 \\
\hline $\begin{array}{l}\text { Personas llegadas entre 2002-2013 que siguen en España en julio de } 2013 \\
\text { (ECH) }\end{array}$ & -3.741 .900 \\
\hline Diferencia: & 2.599 .580 \\
\hline \multicolumn{2}{|l|}{ Total bajas 2002-2013 con destino exterior (EVR): 2.128 .163} \\
\hline $\mathbf{H}_{0}$ mínima: restar todas las bajas 2002-2013 & 471.417 \\
\hline Mínimo \% de subestimación de las bajas en la EVR & $18,13 \% *$ \\
\hline Ho $_{0}$ máxima: restar el $65,3 \%$ de las bajas $2002-2013$ & 1.209 .889 \\
\hline Máximo \% de subestimación de las bajas en la EVR & $46,54 \% * *$ \\
\hline
\end{tabular}

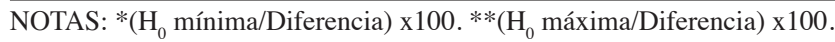

Fuente: INE (EVR y ECH) y elaboración propia.

Si tanto la ECH como las altas de la EVR recogen adecuadamente la realidad, puede apreciarse en la tabla 3 que el intervalo de la horquilla de subestimación de las bajas en la EVR se ha reducido sensiblemente. De una subestimación mínima con el Censo del 40,01\% a un mínimo con la ECH del 18,13\% y de un máximo del 64,01\% con el Censo a un máximo del 46,54\% con la ECH. Y lo que es más interesante, los niveles a los que se mueve el intervalo se han reducido al tiempo en que persiste la recesión económica y sería esperable mayor intensidad en las salidas y, por tanto, mayor subestimación. No es esta una mala noticia para la EVR.

\section{IV.1.2. Y ¿cómo se cumplen las igualdades demográficas? Una sorpresa con la ESMI}

La contabilidad demográfica proporciona una igualdad que permite poner en conexión stocks y flujos de población. El ejercicio que planteamos ahora consiste en añadir al stock inicial de población (Censo de 2011) los flujos internacionales netos en un periodo (EVR 
o ESMI), para verificar si el resultado es similar al stock de población al final del periodo (ECH con fecha de referencia en julio de 2013). Así, se dispone del número de nacidos en el extranjero residentes en viviendas familiares del Censo 2011 (5.649.185 a 1 de noviembre); de los flujos de entradas y salidas internacionales de nacidos en el extranjero desde noviembre de 2011 a junio (incluido) de 2013 de la EVR (y, alternativamente, de la ESMI); de la mortalidad anual entre los extranjeros en el mismo periodo ${ }^{14} \mathrm{y}$, finalmente, del stock de nacidos en el extranjero a 1 de julio de 2013 (5.734.100) que facilita la ECH.

Tabla 4

SUBESTIMACIÓN DE LAS BAJAS DE NACIDOS FUERAA PARTIR DE IGUALDADES DEMOGRÁFICAS (2011-2013)

\begin{tabular}{|c|c|c|c|c|c|}
\hline \multicolumn{2}{|r|}{ Censo de 2011} & 5.649 .185 & & & \\
\hline \multicolumn{2}{|r|}{ Flujos migratorios } & Total & $2011^{*}$ & 2012 & $2013 * *$ \\
\hline \multirow{2}{*}{ EVR } & + Altas & 560.135 & 60.463 & 345.151 & 154.521 \\
\hline & - Bajas & 553.719 & 53.690 & 325.492 & 174.537 \\
\hline \multirow{2}{*}{ ESMI } & + Altas & 453.827 & 53.246 & 283.720 & 116.860 \\
\hline & - Bajas & 680.838 & 59.501 & 389.275 & 232.062 \\
\hline \multicolumn{2}{|r|}{ - Defunciones extranjeros } & 18.618 & 1.748 & 11.288 & 5.582 \\
\hline \multicolumn{2}{|r|}{ Stock estimado EVR } & 5.636 .983 & & & \\
\hline \multicolumn{2}{|r|}{ Stock estimado ESMI } & 5.403 .556 & & & \\
\hline \multicolumn{2}{|r|}{ ECH julio 2013} & 5.734 .100 & & & \\
\hline & ECH-Stock EVR & 97.117 & & & \\
\hline & ECH-Stock ESMI & 330.544 & & & \\
\hline
\end{tabular}

NOTAS: * Noviembre y diciembre; $* *$ Enero a junio.

Fuente: INE (EVR, ESMI, ECH, Censo de 2011 y MNPE) y elaboración propia.

Desde el punto de vista empírico el resultado se muestra en las dos últimas filas de la tabla 4. Y es bien sorprendente. Si se admite que el Censo de 2011, las altas de la EVR y la ECH reflejan bien el fenómeno que estamos estudiando, entonces las 97.117 personas que «faltarían» en julio de 2013 según la ECH corresponderían a falsas bajas en la EVR, quizá a parte de las bajas de oficio dadas por los ayuntamientos ${ }^{15}$. Es decir, que al día de hoy, no habría en la EVR subestimación de las salidas de los nacidos fuera al exterior, más bien lo contrario.

Motivo de preocupación sí debería ser el resultado obtenido a partir del uso de los flujos de la ESMI. Como ya se ha indicado, la ESMI es fruto de la «depuración» de las variaciones residenciales declaradas que hace el INE para mejorar la observación directa del fenómeno demográfico más allá de las simples declaraciones de altas y bajas de la EVR. Pues lo cierto

14 En este caso se incluyen las defunciones, aunque se trate de extranjeros y no de nacidos fuera, ya que se están comparando stocks.

15 Estas bajas no son registradas por los individuos sino por los ayuntamientos cuando no hay constancia oficial de que un empadronado siga residiendo en el municipio. Se explican con detalle más adelante en texto. 
es que ajustando sus flujos al Censo de 2011 y comparando el resultado con el stock estimado por la ECH, deja de computar a más de 330.000 nacidos fuera que todavía estarían residiendo en España a mediados de 2013. Quizá es el momento de revisar los criterios (coeficientes de expansión, factores de ponderación...) utilizados en la ESMI con los que los técnicos del INE intentan trasladar migraciones (movimientos) a migrantes (personas).

\section{IV.1.3. Comparando los recién llegados captados por la EVR y la ECH}

Este ejercicio se va a realizar para el año 2013, ya que es el único en el que, por el momento, la ECH ha arrojado resultados (provisionales y con fecha de referencia en julio de 2013). A los nacidos fuera de España se les pregunta en esta encuesta por la fecha en la que establecieron su residencia en el país por última vez. De este modo, podemos conocer el tiempo que este colectivo lleva residiendo en España. En especial, podemos centrarnos en el stock de nacidos en el extranjero procedentes del extranjero que llevan residiendo en España menos de un año y compararlo con las altas en la EVR. Para ello, hay que adaptar los microdatos de la EVR a los criterios de la ECH; esto es, se ha seleccionado a los nacidos fuera de todas las edades y nacionalidades que llevan residiendo en España menos de 1 año; esto es, con altas entre julio de 2012 y junio de 2013, al ser la fecha de referencia de la ECH el mes de julio de 2013 .

Teóricamente, las altas procedentes del extranjero de la EVR deberían estar ligeramente por encima de los inmigrantes capturados por la ECH, tanto porque en la ECH se pide la última entrada desde el extranjero como porque los que capta son inmigrantes supervivientes hasta el momento de la entrevista. No obstante, la diferencia debería ser relativamente pequeña, ya que la probabilidad del suceso de no emerger en la encuesta (por defunción o emigración) es reducida dado el corto espacio de tiempo entre la llegada y la encuesta. Puede apreciarse en la tabla 5 que esto no se produce: la EVR está casi un $7 \%$ por debajo de la $\mathrm{ECH}^{16}$ o, dicho de otro modo, hay 23.127 inmigrantes de la ECH que no tendrían -todavíasu correspondiente entrada registrada en la EVR. Un número mayor de inmigrantes en la encuesta que el que correspondería a las entradas registradas confirma que, desde luego, la EVR no sobreestima el flujo de inmigración.

No obstante, llama la atención que la diferencia no se distribuye de forma homogénea, independientemente de las características de los inmigrantes. De hecho, sólo es para el sexo femenino cuando la ECH supera a la EVR $(16,6 \%)$ y, especialmente, entre las mujeres en edades de 25 a 44 años ${ }^{17}$. Ante estos resultados las preguntas son inmediatas: ¿las mujeres se inscriben menos en el padrón porque no lo necesitan si lo hacen los cabezas de familia?; ¿hay más tendencia a registrarse entre ciertas nacionalidades por alguna razón y a marcharse

16 Y la diferencia entre la ECH y la ESMI es todavía mayor, del 23\%; lo que supone que se triplica el número de inmigrantes sin registro de alta (más de 78.000).

17 Tampoco desde el punto de vista del país de nacimiento la diferencia es uniforme. Mientras que hay un saldo a favor de la EVR (un exceso de 51.428 personas) en los nacidos en el Reino Unido, en Europa no-UE 27, en África (incluido Marruecos) y en Asia y Oceanía; por el contrario, la ECH encuentra bastantes más efectivos que la EVR (74.755 personas más) si se trata de nacidos en países de la UE-27 distintos del Reino Unido y en el conjunto del continente americano. 
Tabla 5

STOCKS DE NACIDOS FUERA PROCEDENTES DEL EXTRANJERO CON MENOS DE UN AÑO DE RESIDENCIA EN ESPAÑA. ECH Y EVR (JULIO 2012- JUNIO 2013)

\begin{tabular}{|c|c|c|c|c|c|c|c|c|c|}
\hline \multirow[b]{2}{*}{ Edad } & \multicolumn{3}{|c|}{ ECH } & \multicolumn{3}{|c|}{ EVR } & \multicolumn{3}{|c|}{ Diferencia EVR-ECH (\%) } \\
\hline & Total & Hombre & Mujer & Total & Hombre & Mujer & Total & Hombre & Mujer \\
\hline Total & 338.900 & 155.100 & 183.800 & 315.773 & 162.546 & 153.227 & $\begin{array}{l}-23.127 \\
(-6,8 \%)\end{array}$ & $\begin{array}{r}7.446 \\
(4,8 \%)\end{array}$ & $\begin{array}{l}-30.573 \\
(-16,6 \%)\end{array}$ \\
\hline 0-14 años & 42.800 & 21.800 & 21.000 & 40.374 & 21.275 & 19.099 & -2.426 & -525 & -1.901 \\
\hline 15-24 años & 58.200 & 23.000 & 35.200 & 66.163 & 31.214 & 34.949 & 7.963 & 8.214 & -251 \\
\hline 25-34 años & 103.300 & 44.300 & 59.000 & 90.222 & 46.797 & 43.425 & -13.078 & 2.497 & -15.575 \\
\hline 35-44 años & 71.100 & 33.100 & 38.000 & 54.153 & 31.022 & 23.131 & -16.947 & -2.078 & -14.869 \\
\hline 45-54 años & 32.800 & 16.800 & 16.000 & 30.609 & 16.077 & 14.532 & -2.191 & -723 & -1.468 \\
\hline 55-64 años & 13.300 & 8.200 & 5.000 & 19.536 & 9.086 & 10.450 & 6.236 & 886 & 5.450 \\
\hline 650 más & 17.400 & 7.900 & 9.600 & 14.716 & 7.075 & 7.641 & -2.684 & -825 & -1.959 \\
\hline
\end{tabular}

Fuente: INE (ECH y EVR) y elaboración propia.

a los pocos meses desde la llegada y, por eso, no emergen en la posterior $\mathrm{ECH}^{18}$ ? o ¿por qué los rumanos, alemanes, colombianos, ecuatorianos o bolivianos habrían de tener menor tendencia a inscribirse en el registro?

Aunque esas diferencias en parte quedaran justificadas por razones concretas, no hay que perder de vista la posibilidad de que -como en la EPA- la estructura familiar, la distribución por sexos y la situación en el mercado laboral de los diferentes colectivos de nacidos fuera recién llegados, hagan que sea más probable «encontrar» a unos que a otros a la hora de la encuesta y que, por tanto, se generen sesgos que provocan que los afloramientos de los llegados recientemente presenten este carácter irregular. Si esto es así, en la recién implementada ECH se debería revisar con cuidado los resultados de ciertas preguntas, como las del tiempo de residencia.

\section{IV.2. La Encuesta de Movilidad Laboral y Geográfica (EMLG)}

Hace ya cierto tiempo que los institutos nacionales de estadística de países de nuestro entorno (Dinamarca, Finlandia, Noruega o Suecia) llevan enlazando la información individual procedente de distintas fuentes estadísticas. Esto es lo que hace el INE (2013) por primera vez con la EMLG. Se trata de una operación estadística en la que se utiliza la información proporcionada por la muestra de la EPA del primer trimestre de cada año vinculándola a los datos de variaciones residenciales registrados en la Base Padronal del INE. De este modo, se cubre la laguna en la información estadística sobre migraciones que provocó la entrada en vigor en 1985 de la Ley Reguladora de las Bases del Régimen Local. A resultas de la cual y en virtud del principio constitucional de la protección de la intimidad, las hojas padronales dejaron de recabar información, entre otros temas, de la situación en el mercado laboral de las personas con cambios de residencia.

18 Por ejemplo, el caso de los británicos que hacen turismo sanitario. 
La EMLG tiene periodicidad anual y, por el momento, se ha divulgado la serie 20102014, con fecha de referencia en el primer trimestre de cada año. Para elaborar los datos anuales, el procedimiento comienza con la localización de los mayores de 15 años encuestados por la EPA en la correspondiente Base Padronal. Una vez identificados, se obtiene la fecha de la última variación residencial anterior a la semana de referencia de la EPA, que es la que sirve para determinar el tiempo que el individuo lleva residiendo en el municipio actual ${ }^{19}$. A efectos de lo anterior, cuando en la Base Padronal no hay información del municipio o país de procedencia anterior del encuestado (caso de las altas por omisión), el INE ha optado por imputar como tal el municipio o país de nacimiento de la persona. Y, por último, conviene recordar que, una vez hecha la depuración y para la submuestra resultante, el INE no lleva a cabo ajustes de calibrado de los factores de elevación, que siguen siendo los utilizados en la $\mathrm{EPA}^{20}$.

A partir de la información de la EMLG, se puede conocer el número de personas de 16 y más años que, procediendo del exterior, llevan menos de un año residiendo en su actual municipio $^{21}$, para cada uno de los años disponibles. Esto nos permite comparar de forma muy ajustada con la EVR. En concreto, se han extraído de los ficheros anuales $(t)$ de microdatos de esta última, las altas de población de 16 y más años procedentes del exterior, que se comparan con los stocks estimados en el primer trimestre del año siguiente $(t+l)$ proporcionados por la EMLG.

En la tabla 6 puede apreciarse que, por una vez, en los años contemplados las altas de la EVR (1.799.907) sí superan a los inmigrantes supervivientes captados por la EMLG (868.937). Sin embargo, para comparar correctamente habría que descontar de la cifra total de altas anuales, al menos, los movimientos múltiples (migrantes que, procedentes del exterior hacen una o varias migraciones interiores posteriores en el mismo año) y las bajas al exterior de los llegados a lo largo del mismo año ${ }^{22}$; esto es, comparar con las altas netas de la EVR. En el caso de las migraciones múltiples, con apoyo en los microdatos de la EVR se ha realizado una estimación de mínimos a través de la agregación de las inscripciones en el mismo año identificables como del mismo individuo ${ }^{23}$, y con coheren-

19 En INE (2014h), que es donde se describe la metodología de esta estadística, se indica que hay una depuración exhaustiva sobre el enlace de identificación de las personas de 16 o más años incluidas en la muestra EPA. Es importante señalar a este respecto que la tasa de enlace entre los encuestados mayores de 16 años de la muestra EPA y la Base Padronal se sitúa en torno al 99,4\% -ver punto 14.3 «Errores ajenos al muestreo» en INE (2014h)-.

20 Ver en INE (2014h) punto 20.6 «Ajuste». La motivación de esta decisión -que, obviamente, tiene consecuencias para las estimaciones- no se ha encontrado en el documento metodológico.

21 En realidad, trabajamos con el resultado de estimar este colectivo EMLG a partir de la información proporcionada por el INE hasta el momento: ocupados y parados de 16 y más años, tasas de actividad por lugar de nacimiento, tiempo de estancia en el actual municipio de residencia y lugar de procedencia.

22 En la metodología de la EMLG se advierte de que las altas por omisión en la Base Padronal no necesariamente se han eliminado de la submuestra EPA, por eso de las altas de la EVR no hemos restado las altas por omisión (de extranjeros desde, normalmente, el extranjero), aunque se sepa que no se producen en el momento de la llegada, sino que se suele tratar de personas que ya estaba en España con anterioridad.

23 Las variables que, teóricamente, identificarían al mismo individuo con varias altas son: sexo, lugar de nacimiento (provincia y municipio o país, si es en el extranjero), día, mes y año de nacimiento y nacionalidad.

Sin embargo, la estructura de los ficheros de microdatos para el año 2013 ha cambiado, dejando de incluirse tanto el día de nacimiento como el de la variación residencial. Por eso, las migraciones repetidas de 2013 se han levantado, añadiendo el porcentaje que en 2012 supone la diferencia entre estimar repetidas con todas las variables de identificación y hacerlo omitiendo los días de nacimiento y de variación residencial. 
cia espacial y temporal. Puede cifrarse en un mínimo entre 6.000 y 10.000 las altas que habría que descontar del total anual procedentes del exterior, por tratarse de personas que han hecho un movimiento interior posterior y que, de ser submuestra EMLG, se les habrá asignado desde la Base Padronal una última residencia en España. Se trata, en todo caso, de un mínimo ya que sólo se han descontado las segundas altas interiores, pero no otras sucesivas en el mismo año.

Tabla 6

STOCKS DE INMIGRANTES PROCEDENTES DEL EXTRANJERO CON 16 AÑOS O MÁS AÑOS DE EDAD Y MENOS DE UNAÑO DE RESIDENCIA EN ESPAÑA. EMLG Y EVR (2009-2013)

\begin{tabular}{|c|c|c|c|c|c|c|c|c|c|}
\hline & \multirow[b]{2}{*}{$\begin{array}{l}\text { EMLG* }^{*} \\
\text { (1) }\end{array}$} & \multirow[b]{2}{*}{$\begin{array}{l}\text { Altas } \\
\text { EVR }\end{array}$} & \multicolumn{5}{|c|}{ Estimaciones microdatos EVR: } & \multirow[b]{2}{*}{$\begin{array}{c}\text { Diferencia } \\
\text { (2)-(1) }\end{array}$} & \multirow[b]{2}{*}{$\begin{array}{c}\text { Diferencia } \\
\text { sobre (2) } \\
(\%)\end{array}$} \\
\hline & & & $\begin{array}{c}\text { Repetidas } \\
\text { destino } \\
\text { interior }\end{array}$ & $\begin{array}{c}\text { Repetidas } \\
\text { destino } \\
\text { exterior }\end{array}$ & $\begin{array}{c}\text { BC en t } \\
\text { de altas } \\
\text { en } t\end{array}$ & $\begin{array}{c}\text { BII en } t \\
\text { de altas } \\
\text { en } t\end{array}$ & $\begin{array}{c}\text { Altas } \\
\text { netas (2) }\end{array}$ & & \\
\hline 2009 & 218.182 & 419.063 & 10.350 & 5.187 & 30.459 & 15.697 & 357.370 & 139.188 & 38,9 \\
\hline 2010 & 205.798 & 392.955 & 9.412 & 5.313 & 28.352 & 16.497 & 333.381 & 127.582 & 38,3 \\
\hline 2011 & 214.378 & 385.435 & 9.541 & 5.790 & 26.120 & 24.086 & 319.898 & 105.519 & 33,0 \\
\hline 2012 & 126.698 & 314.189 & 8.338 & 5.156 & $25.272^{* *}$ & $24.234^{* *}$ & 251.188 & 124.490 & 49,6 \\
\hline 2013 & 103.880 & 288.265 & 5.934 & 5.776 & $24.413^{* *}$ & $25.782^{* *}$ & 226.359 & 122.478 & 54,1 \\
\hline Suma & 868.937 & 1.799 .907 & 43.575 & 27.222 & 134.616 & 106.297 & 1.488 .195 & 619.258 & 41,6 \\
\hline
\end{tabular}

NOTAS: ${ }^{*}$ La fecha de referencia de la EMLG es el primer trimestre año siguiente; ${ }^{* *}$ Estimación mediante proyección logarítmica de la tendencia.

Fuente: EMLG y EVR (INE) y elaboración propia.

Como reconoce el INE, las bajas por salida al exterior es el punto débil de la EVR y, también, de la tabla 6. En nuestro caso tenemos que encontrar la forma de sustraer de las entradas anuales las bajas correspondientes al mismo año de alta, porque lo deseable es comparar el flujo neto anual de la EVR con los inmigrantes teóricamente supervivientes al final de cada año de la EMLG. Para averiguar cuántas de las altas se han convertido en bajas en el mismo año agregaremos dos componentes. Por un lado, si en los microdatos enlazados de la EVR se exige que el segundo movimiento sea de salida al exterior, se obtiene que habría que restar de las altas más de 5.000 bajas anuales por emigración al extranjero de personas que por no estar ya en España, lógicamente, nunca habrán llegado a ser entrevistadas en la EMLG. A ese componente hay que sumar un segundo bloque que puede, al menos, aproximarse. Se trata de la parte de las bajas de oficio por inclusión indebida (BII) y por caducidad (BC) que correspondiendo al mismo año del alta y que se conocen con posterioridad. Las BII son las bajas de extranjeros inscritas por los ayuntamientos por emigración con destino al exterior en las que se desconoce el país de destino del emigrante. Las BII se incluyen en la EVR desde 2004, y desde 2008 se utilizan de forma intensiva para des-registrar a los extranjeros comunitarios o a los que tienen permiso de residencia permanente, si no renuevan su inscripción padronal cada cinco años si están inscritos en el Registro Central de Extranjeros (RCE) y cada dos si no lo están. Precisamente en 2013, cuando se cumplen cinco años desde 
la entrada en vigor del acuerdo del Consejo de Empadronamiento de 2008 que da lugar a la intensificación mencionada, estas bajas se han elevado al 56\% desde el 33\% del total de bajas de extranjeros en 2009. Por su parte, las BC que se incorporan en la EVR a partir de 2006 consisten en bajas inscritas por los ayuntamientos de Extranjeros No Comunitarios Sin Autorización de Residencia Permanente-ENCSARP- que no han renovado su inscripción padronal en los dos últimos años. En 2009 representaban el 54\% de las bajas de extranjeros y en 2013 se han reducido hasta el $32 \%$.

A efectos oficiales, estas personas ya no se encuentran en el país, pero el problema de estas bajas es que sólo se conocen a posteriori, aunque sí se puede inferir el año en que se produjo la última alta. Para las $\mathrm{BC}$ del año $t$ las altas correspondientes se registraron en el año $t$-2, del mismo modo que si se trata de BII de extranjeros NO-ENCSARP no inscritos en el RCE. Si se trata de extranjeros NO-ENCSARP sí inscritos en el RCE, entonces el alta última se produjo en $t$-5. Suponiendo que en la mayor parte de casos se trata de altas por entradas desde el exterior y, a falta de otra hipótesis, se ha optado por recurrir a la estimación del $25 \%$ de las altas que hace el propio INE en 2009 en relación con la asignación temporal de las bajas por caducidad para el cálculo de sus Estimaciones de Población Actual24; dicho de otro modo, supondremos que al menos el $25 \%$ de las $\mathrm{BC}$ y de las $\mathrm{BII}^{25}$ de las personas que han registrado un alta vuelve al exterior en el mismo año de su llegada. Descontadas estas bajas, la diferencia con la cifra de inmigrantes que proporciona la ELMG equivale entre un mínimo del $33 \%$ y un máximo del $54 \%$ de las altas netas anuales (tabla 6$)^{26}$.

¿Quiere decir esto que la EVR subestima las bajas anuales al exterior en una horquilla entre el $33 \%$ y el 54\% de las altas? No, con toda seguridad es un intervalo que se sitúa muy por encima del real, porque la EMLG subestima a la población con movilidad. De hecho, este es un problema que se deriva de la propia $\mathrm{EPA}^{27}$. Por un lado, porque el diseño muestral de la EPA tiende a subestimar fuertemente las llegadas recientes de los inmigrantes. No hay más que comprobar cómo la cohorte de inmigrantes de entrada en un año concreto aumenta desproporcionadamente en la EPA cuando se la estima al año siguiente y sucesivos. Esto sucede incluso en plena crisis: las entradas de extranjeros procedentes del exterior a lo largo de 2008 aumentan un 49\% si se computan desde 2009, las de 2009 un 57\% si se computan desde 2010 y así sucesivamente hasta las entradas en 2012 que aumentan otro 57\% si se evalúan un año más tarde. Por tanto, si la EPA no capta bien la inmigración reciente, su muestra, que es la que se utiliza para la EMLG, tampoco.

24 En concreto, señala que: «Seguidamente, al total de bajas por caducidad confirmadas resultante de las estimaciones anteriores, se le supone un perfil de salida del país a partir del último contacto con el ayuntamiento de tres años (25\% para ese año del último contacto, 50\% para el siguiente, y 25\% para el último)». Ver INE (2014i:27).

A modo de ejemplo, de las 104.480 BC de mayores de 15 años del año 2013 que corresponden a altas efectuadas a lo largo del año 2011, el 25\% (26.120) se trataría de retornos al exterior en el mismo año 2011.

25 Solo se van a tener en cuenta las BII de los no registrados en el RCE (que se producen a los dos años de la última inscripción), suponiendo que son la mitad de los NO-ENCSARP.

26 Este ejercicio no se puede hacer con la ESMI, pues en sus ficheros de microdatos para cada individuo con información incompleta no hay un único registro sino varios, dependiendo de las probabilidades asignadas a cada posible país de nacimiento, de nacionalidad, de origen o destino en estos casos en que no constan. Esta estructura de los ficheros con registros ponderados hace que sea muy difícil la selección de individuos con alta y baja exterior en el mismo año.

27 Ver Martí y Ródenas (2012a) y Ródenas y Martí (2013). 
Por otro lado, la EPA no es coherente con sus propias cifras de movilidad: la población de nacionalidad extranjera que lleva menos de un año residiendo en España es, sorprendentemente, superior a la cifra de las personas de cualquier nacionalidad que un año atrás residía en el exterior. Como se aprecia en la tabla 7, la diferencia media se sitúa en un 22,4\%. Como la pregunta acerca del año de llegada a España sólo se realiza a los extranjeros, los datos de movilidad que se utilizan en la EPA para armar la EMLG tienen que ser los de la submuestra que el INE utiliza para estimar las variables estructurales anuales. Si ya sabemos que éstos no reflejan adecuadamente la movilidad reciente, los individuos de la submuestra para los que se requiera hacer el enlace geográfico de la EPA a la Base Padronal serán pocos, insuficientes para captar la verdadera intensidad de la movilidad. Por tanto, la EMLG estará sesgada a la baja en cuanto a las migraciones recientes, al menos seguro que un $22,4 \%$. De acuerdo con eso, si aumentamos el número de inmigrantes de la EMLG en ese porcentaje, entonces la horquilla de subestimación de las bajas de la EVR disminuye a los niveles de entre el $18 \%$ y el 43,8\% de las altas en el mismo año; o, en media para el período 2009-2013, al 28,5\%.

Tabla 7

INMIGRANTES PROCEDENTES DEL EXTERIOR (MILES DE PERSONAS). EPA (2006-2013)

\begin{tabular}{|l|c|c|c|c|c|c|c|c|c|}
\hline & $\mathbf{2 0 0 6}$ & $\mathbf{2 0 0 7}$ & $\mathbf{2 0 0 8}$ & $\mathbf{2 0 0 9}$ & $\mathbf{2 0 1 0}$ & $\mathbf{2 0 1 1}$ & $\mathbf{2 0 1 2}$ & $\mathbf{2 0 1 3}$ & Suma \\
\hline $\begin{array}{l}\text { Personas que han } \\
\text { cambiado de residencia } \\
\text { hace un año procedentes } \\
\text { del exterior (Variable } \\
\text { anual de submuestra) }\end{array}$ & 251,6 & 215,9 & 211,9 & 144,7 & 88,0 & 98,7 & 77,1 & 72,1 & 1.160 \\
\hline $\begin{array}{l}\text { Extranjeros por tiempo } \\
\text { de residencia en España } \\
\text { (menos de un año) }\end{array}$ & 322,0 & 313,7 & 245,5 & 146 & 107,1 & 113,8 & 89,2 & 82,7 & 1.420 \\
\hline \multicolumn{1}{|c|}{ Diferencia (\%) } & $-28,0$ & $-45,3$ & $-15,9$ & $-0,9$ & $-21,7$ & $-15,3$ & $-15,7$ & $-14,7$ & $-22,4$ \\
\hline
\end{tabular}

Fuente: EPA y elaboración propia.

\section{CONCLUSIONES}

Las discrepancias entre las cifras migratorias de las estadísticas españolas no nos están permitiendo confirmar cuántos inmigrantes se han marchado y cuántos quedan de los que llegaron antes de la fase recesiva del ciclo económico. Dada la reciente presentación de nuevas estadísticas migratorias elaboradas por el INE, el trabajo se ha centrado en analizar los resultados que arrojan estas fuentes sobre las actuales migraciones internacionales y utilizarlas para precisar el grado de fiabilidad de la, ya tradicional, EVR.

El primer grupo de resultados se refiere a la cuantificación de la subestimación de las bajas en la EVR por cohorte anual de entrada. En primer lugar, una vez verificado que el Censo de 2011 no tiene los problemas de infravaloración de la inmigración reciente que sí presentaba el Censo de 2001, se utilizan sus datos para aproximar una primera horquilla al grado de subestimación de las bajas al exterior de la EVR de los nacidos fuera para las cohortes de entrada en el período 2002-2011. A la suma de las diferencias anuales entre las altas registradas y los inmigrantes supervivientes en 2011, se le ha descontado las salidas, 
estableciendo dos hipótesis para imputar a las altas las bajas sí registradas en el período. Así, la EVR podría dejar de haber registrado entre el 40,01\% de las bajas y el 64,01\%. En segundo lugar, se ha realizado un ejercicio similar pero tomando como base la nueva ECH y con fecha de referencia más actual, julio de 2013, que proporciona un segundo intervalo: la EVR subestimaría entre un mínimo equivalente al 18,13\% de las altas y un máximo equivalente al 46,54\%. Es de resaltar que la banda de subestimación descienda respecto de 2011 al tiempo que persiste la recesión económica y sería esperable mayor intensidad en las salidas y, por tanto, mayor subestimación. Y, finalmente, la última comprobación se ha realizado a partir de la EMLG, que utiliza la información proporcionada por la muestra de la EPA vinculándola a los datos de variaciones residenciales registrados en la Base Padronal del INE. Se ha comparado el número de personas de 16 y más años que, procediendo del exterior, llevan menos de un año residiendo en su actual municipio de esta estadística con las altas netas (ajustando las BC y las BII) de la EVR para el periodo 2009-2013. Teniendo también en cuenta que la subestimación de la movilidad reciente que afecta a la EPA se traslada a la EMLG y que puede consistir en, al menos, un 22,4\%, entonces la última horquilla de subestimación de las bajas de la EVR se situaría entre el $18 \%$ y el $43,8 \%$ de las altas en el mismo año. Valorando las tres horquillas, parece razonable concluir con que en la EVR podría estar dejándose de contabilizar como bajas en torno a un tercio de las altas de cada año.

Adicionalmente, en el trabajo se han obtenido otros dos resultados que, desde el punto de vista de la calidad de las nuevas fuentes estadísticas, deberían ser objeto de reflexión. Por un lado, es motivo de preocupación que para un año concreto y reciente, 2013, no se encuentre que las altas procedentes del extranjero de la EVR estén, como deberían, ligeramente por encima de los inmigrantes capturados por la ECH. En concreto, la ECH fija en 338.900 los nacidos fuera procedentes de extranjero con menos de un año de residencia en España en julio de 2013 y, sin embargo, en la EVR sólo hay registradas 315.773 inmigraciones para este colectivo entre julio de 2012 y junio de 2013; esto es, casi un $7 \%$ menos. Esto confirma que, desde luego, la EVR no sobreestima el flujo de inmigración. Pero, el hecho de que esa diferencia no se distribuya de forma homogénea o independiente de las características de los inmigrantes debería ser una buena razón para considerar la posibilidad de que en la recién implementada ECH hubiera algún tipo de sesgo. Que el exceso se concentre exclusivamente en el sexo femenino y en las edades de 25 a 44 años, recuerda los problemas de diseño muestral generados cuando la estructura familiar, la distribución por sexos o la situación en el mercado laboral de los diferentes colectivos, hacen que sea más probable «encontrar» a unos que a otros a la hora de la encuesta. Y que, por tanto, se generen sesgos que provocan que los afloramientos de los llegados hace escaso tiempo (saldrán muchos si son recién llegados en desempleo) presenten este carácter irregular. Si esto es así, en la ECH se debería revisar con cuidado los resultados de preguntas como las del tiempo de residencia.

Finalmente, y dado que la contabilidad demográfica proporciona una igualdad que permite poner en conexión stocks y flujos de población, en el trabajo se ha añadido al stock inicial de nacidos en el extranjero del Censo de 2011 los flujos internacionales netos EVR/ ESMI entre enero de 2012 y junio de 2013. El resultado es que faltarían 97.117 personas según la EVR y, lo que es peor, más de 330.000 según la ESMI, para igualarse al stock en julio de 2013 proporcionado por la ECH. Si se asume que no hay errores en los stocks inicial 
y final y que las altas reflejan la realidad, entonces la diferencia no puede más que corresponder a falsas bajas en la EVR y en la ESMI, quizá a que parte de las bajas de oficio dadas por los ayuntamientos, BC y BII, no son tales. Dicho de otro modo, al día de hoy no habría en la EVR subestimación de las salidas al exterior de los nacidos fuera, más bien lo contrario. Motivo de preocupación sí debería ser el resultado obtenido a partir del uso de los flujos de la ESMI, de la «depuración» de las variaciones residenciales, pues ahí casi se triplica la diferencia y eso no puede ser más que consecuencia de los criterios (coeficientes de expansión, factores de ponderación, etc.) con los que trabajan los técnicos del INE.

\section{REFERENCIAS BIBLIOGRÁFICAS}

DE CASTRO, M. A. (2004): «Fuentes estadísticas sobre la inmigración», Economistas, 99, pp. 128-145.

DOMINGO, A. SABATER, A. (2013): «Emigración marroquí desde España en contexto de crisis», Revista Internacional de Estudios Migratorios, 3 (1), pp. 29-60.

GARRIDO, L. (2004): «Para cuantificar a los extranjeros», Economistas, 99, pp. 28-37.

INE (2009): «Comentarios al artículo ¿Son fiables los datos de migraciones del Censo de 2001?», Revista de Economía Aplicada, vol.17 (51), pp. 141-146.

INE (2013): Estadística de Movilidad Laboral y Geográfica (Explotación Estadística de la Encuesta de Población Activa y de la Base Padronal del INE). Metodología y descripción general de la operación.

INE (2014a): Estadística de Variaciones Residenciales. Metodología. Disponible en: http:// www.ine.es/daco/daco42/migracion/ notaevr.htm, consultado en noviembre de 2014.

INE (2014b): Estadística de Variaciones Residenciales. Informes Metodológicos Estandarizados. Disponible en: http://www.ine.es/dynt3/metadatos/es/RespuestaDatos. htm?oe $=30307$

INE (2014c): Estadística de Migraciones. Metodología.

INE (2014d): Metodología de cálculo de las cifras de población censal, abril. Disponible en: http://www.ine.es/CDINEbase/consultar.do?mes=\&operacion=Censos+de+Poblaci\%F3n $+\mathrm{y}+$ Viviendas\&id_oper=Ir

INE (2014e): Informes Metodológicos Estandarizados. Censo de población y Viviendas 2011. Disponible en http://www.ine.es/dynt3/metadatos/es/RespuestaDatos.htm?oe=30243

INE (2014f): Así se hicieron los Censos 2001. Disponible en: http://www.ine.es/censo2001/ internet.htm

INE (2014g): Encuesta Continua de Hogares. Metodología. Disponible en: http://www.ine. es/inebaseDYN/ech30274/docs/meto_ech.pdf

INE (2014h): Informe Metodológico Estandarizado. Estadística de Movilidad Laboral y Geográfica. Disponible en: http://www.ine.es/dynt3/metadatos/es/RespuestaDatos. htm?oe $=30209$

INE (2014i): Estimaciones de la Población Actual. Metodología detallada (Metodología vigente hasta el 1 de enero de 2009). Disponible en: http://www.ine.es/daco/daco43/ epoba/metodo.pdf

MARTÍ, M. y RÓDENAS, C. (2004): «Migrantes y migraciones: de nuevo la divergencia en las fuentes estadísticas», Estadística Española, vol. 46, n 156, pp. 293-321. 
MARTÍ, M. y RÓDENAS, C. (2007): «Migration Estimation based on the Labour Force Survey: An EU-15 Perspective», International Migration Review, vol. 41 (1), pp. 101-126.

MARTÍ, M. y RÓDENAS, C. (2012a): «Measuring international migration through sample surveys: some lessons from the Spanish case», Population-E, 67 (3), pp. 435-464. ISSN: 1634-2941.

MARTÍ, M. y RÓDENAS, C. (2012b): «Reemigrar en España: una aproximación a sus determinantes», Investigaciones Regionales, $\mathrm{n}^{\circ} 22$, pp. 105-128.

POULAIN, M.; PERRIN, N. y SINGLETON, A.T. (Eds.) (2006): THESIM. Towards Harmonised European Statistics on International Migration, Louvain: Presses Universitaires de Louvain.

RÓDENAS, C. y M. MARTÍ (1997): «¿Son bajos los flujos migratorios en España?», Revista de Economía Aplicada, $\mathrm{n}^{\circ}$ 15, vol. V, pp. 155-171.

RÓDENAS, C. y MARTÍ, M. (2006): «Reinterpretando el crecimiento de la movilidad de España: la población extranjera y las migraciones repetidas», Cuadernos Aragoneses de Economía, vol. 16 (1), pp. 37-59.

RÓDENAS, C. y MARTÍ, M. (2009a): «¿Son fiables los datos de migraciones del Censo de 2001?», Revista de Economía Aplicada, vol. 17 (50), pp. 97-118.

RÓDENAS, C. y MARTÍ, M. (2009b): «Observaciones a los comentarios del INE al artículo ¿Son fiables los datos de migraciones del Censo de 2001?», Revista de Economía Aplicada, vol. 17 (51), pp.147-156.

RÓDENAS, C. y MARTÍ, M. (2009c): «Estimating False Migrations in Spain», PopulationE, vol. 64 (2), pp. 361-376.

RÓDENAS, C. y MARTÍ, M. (2013): «La nueva Estadística de Migraciones: una buena elección por parte del INE», Revista Española de Investigaciones Sociológicas, $\mathrm{n}^{\circ} 143$, pp. 113-120.

SUSINO, J. (2011): «La evolución de las migraciones interiores en España: una evaluación de las fuentes demográficas disponibles», Papers, nº 96 (3), pp. 853-881.

SUSINO, J. (2012): «Fuentes demográficas para el estudio de la migración en España», Revista Interdisciplinar da Mobilidade Humana, vol. XX (39), pp. 51-76. 